\title{
RELAÇÃO ENTRE CONCENTRAÇÕES FOLIARES DE CARBOIDRATOS SOLÚVEIS TOTAIS E TOLERÂNCIA AO FRIO EM DIFERENTES ESPÉCIES DE Eucalyptus spp.
}

\section{RELATIONSHIP BETWEEN CONCENTRATIONS OF TOTAL SOLUBLE CARBOHYDRATES IN THE LEAVES AND COLD TOLERANCE IN DIFFERENT SPECIES OF Eucalyptus spp.}

\author{
Mireli Moura Pitz Floriani ${ }^{1}$ Cristiano André Steffens ${ }^{2}$ Djalma Miler Chaves ${ }^{3}$ \\ Cassandro Vidal Talamini do Amarante ${ }^{4}$ Tiago Georg Pikart ${ }^{5}$ Maitê dos Santos Ribeiro ${ }^{6}$
}

\begin{abstract}
RESUMO
O objetivo deste trabalho foi avaliar o efeito da rustificação sobre a tolerância ao frio em Eucalyptus dunnii Maiden, Eucalyptus benthamii Maiden \& Cambage, Eucalyptus grandis W. Hill ex Maiden e Eucalyptus saligna Sm., através da quantificação dos teores foliares de carboidratos solúveis totais e de prolina. Mudas das quatro espécies (com três meses de idade e cerca de $50 \mathrm{~cm}$ de altura) foram submetidas a dois períodos de rustificação ( 0 e 21 dias de exposição a temperaturas diurnas de $5{ }^{\circ} \mathrm{C}$ e noturnas de $\left.1{ }^{\circ} \mathrm{C}\right)$, com fotoperíodo controlado de 12 horas. Ao final de cada período de rustificação, as mudas foram submetidas durante 3 horas a três gradientes de temperatura abaixo de zero $\left(-2{ }^{\circ} \mathrm{C},-5^{\circ} \mathrm{C}\right.$ e $\left.-8{ }^{\circ} \mathrm{C}\right)$. Foram feitas avaliações das concentrações foliares de carboidratos solúveis totais e de prolina, da temperatura letal de $50 \%\left(\mathrm{TL}_{50}\right)$ e do índice de dano ao frio. Não foi identificada a presença de prolina nos tecidos foliares das espécies avaliadas. No entanto, em mudas não rustificadas, observou-se diferenças entre as espécies quanto à concentração foliar de carboidratos, sendo maior em Eucalyptus benthamii, intermediária em Eucalyptus dunnii e Eucalyptus grandis, e menor em Eucalyptus saligna. A rustificação causou um aumento na concentração foliar de carboidratos de 2,9; 2,5; 2,8 e 1,3 vezes em Eucalyptus dunnii, Eucalyptus benthamii, Eucalyptus saligna e Eucalyptus grandis, respectivamente. Em mudas que não foram submetidas à rustificação, a $\mathrm{TL}_{50}$ foi mais baixa em Eucalyptus benthamii, intermediária em Eucalyptus dunnii e Eucalyptus grandis, e mais alta em Eucalyptus saligna. No entanto, com a rustificação das plantas não houve diferenças entre as espécies quanto à $\mathrm{TL}_{50}$. Observou-se que a $\mathrm{TL}_{50}$ diminuiu com a rustificação, exceto no Eucalyptus benthamii. Houve correlação negativa entre a concentração foliar de carboidratos solúveis totais e a $\mathrm{TL}_{50}$ nas espécies avaliadas. Os dados obtidos mostram que a concentração foliar de carboidratos solúveis totais pode ser utilizada como indicador de tolerância ao frio em espécies de Eucalyptus.
\end{abstract}

Palavras-chave: temperatura letal de $50 \%\left(\mathrm{TL}_{50}\right)$; aclimatação; temperatura negativa; dano por frio.

\section{ABSTRACT}

This work was carried out to evaluate the effects of the rustification on cold tolerance in Eucalyptus dunnii Maiden, Eucalyptus benthamii Maiden \& Cambage, Eucalyptus grandis W. Hill ex Maiden, and Eucalyptus saligna Sm., by quantifying leaf contents of total soluble carbohydrates and proline. Seedlings (three months

1. Bióloga, Msc., Empresa Klabin S.A., Av. Olinkraft, 6602, CEP 88540-000, Otacílio Costa (SC). mmpitz@klabin.com.br

2. Engenheiro Agrônomo, Dr., Professor Associado do Departamento de Agronomia, Centro de Ciências Agroveterinárias, Universidade do Estado de Santa Catarina, Av Luiz Camões, 2090, Conta Dinheiro, CEP 88520-000, Lages (SC). steffens@cav.udesc.br

3. Engenheiro Agrônomo, PhD., Consultor Coorporativo de Pesquisas Florestais, Empresa Klabin S.A., Av. Olinkraft, 6602, CEP 88540-000, Otacílio Costa (SC).dmchaves@klabin.com.br

4. Engenheiro Agrônomo, PhD., Professor Associado do Departamento de Agronomia, Centro de Ciências Agroveterinárias, Universidade do Estado de Santa Catarina, Av Luiz Camões, 2090, Conta Dinheiro, CEP 88520-000, Lages (SC). amarante@cav.udesc.br

5. Engenheiro Florestal, Mestrando em Entomologia do Departamento de Biologia Animal, Universidade Federal de Viçosa, Av. P. H.. Rolfs, s/n, Campus Universitário, CEP 36570-000 Viçosa (MG). tiago.florestal@gmail.com

6. Engenheira Florestal, Rua Lages 102, Ap. 110, Centro, CEP 89520-000, Curitibanos (SC). maiteribeiro@yahoo.com.br

Recebido para publicação em 16/12/2010 e aceito em 3/10/2011 
old and about $50 \mathrm{~cm}$ height) of these species were submitted to two periods of rustification (zero and 21 days exposure to day/night temperatures of $5{ }^{\circ} \mathrm{C} / 1{ }^{\circ} \mathrm{C}$ ), with photoperiod of 12 hours. After each period of rustification, the seedlings were submitted, for 3 hours, to three temperatures below $0{ }^{\circ} \mathrm{C}\left(-2{ }^{\circ} \mathrm{C},-5^{\circ} \mathrm{C}\right.$, and $\left.-8^{\circ} \mathrm{C}\right)$. The seedlings were then assessed for leaf contents of total soluble carbohydrates and proline, lethal temperature of $50 \%\left(\mathrm{LT}_{50}\right)$, and cold damage index. Proline was not detected in the leaves of all species. However, in non-rustified seedlings, there were differences between the species regarding to the foliar contents of carbohydrates, with the highest values in Eucalyptus benthamii, intermediary in Eucalyptus dunnii and Eucalyptus grandis, and the lowest in Eucalyptus saligna. The rustification treatment increased leaf concentration of total soluble carbohydrates by 2.9, 2.5, 2.8, and 1.3 in Eucalyptus dunnii, Eucalyptus benthamii, Eucalyptus saligna and Eucalyptus grandis, respectively. In the seedlings not submitted to the rustification, the $\mathrm{LT}_{50}$ value was lower in Eucalyptus benthamii, intermediary in Eucalyptus dunnii and Eucalyptus grandis, and higher in Eucalyptus saligna. However, in rustified seedlings the $\mathrm{LT}_{50}$ was no different among the species. Rustification reduced the $\mathrm{LT}_{50}$, except in Eucalyptus benthamii. There was a negative correlation between leaf concentration of total soluble carbohydrates and the $\mathrm{LT}_{50}$, considering all Eucalyptus species evaluated. The results show that leaf concentration of total soluble carbohydrates can be used as an indicator of cold tolerance in species of Eucalyptus.

Kewwords: lethal temperature of $50 \%\left(\mathrm{LT}_{50}\right)$; acclimatization, sub-zero temperature; cold damage.

\section{INTRODUÇÃO}

A ocorrência de geadas é um dos principais fatores climáticos que limitam a expansão dos plantios comerciais de Eucalyptus na região Sul do Brasil. Segundo Higa et al. (1994), as geadas registradas em 1972 causaram, em algumas regiões, perdas de até $100 \%$ das plantas em talhões de espécies florestais suscetíveis. Os efeitos potencialmente prejudiciais de temperaturas abaixo de $0{ }^{\circ} \mathrm{C}$ na estrutura das células de plantas têm sido extensivamente descritos (PALTA et al., 1993; STEPONKUS et al., 1993). Em espécies de Eucalyptus, temperaturas próximas ou abaixo de $0{ }^{\circ} \mathrm{C}$ ocasionam danos que podem levar à perda da área foliar, limitando e modificando o crescimento, podendo causar até a morte das plantas (FILHO et al., 2005).

Os danos nas plantas são causados pelo congelamento do líquido presente nos espaços intracelulares, que causa a ruptura de membranas por compressão e extravasamento irreversível do citoplasma. Segundo Higa et al. (1994), a formação de gelo nos tecidos normalmente ocorre em temperaturas abaixo de $-3{ }^{\circ} \mathrm{C}$ ou $-4{ }^{\circ} \mathrm{C}$. De acordo com esses autores, quanto menor for a adaptação da planta ao abaixamento de temperatura, mais severo será o dano causado pelo congelamento.

Higa et al. (2000) salientam que a escolha da espécie adequada é um dos principais itens para o sucesso do reflorestamento, sendo que, entre os vários fatores que influenciam na tomada de decisão sobre a espécie a ser plantada, destacam-se a finalidade do plantio, o solo e o clima do local. Com relação às espécies de Eucalyptus, a seleção, em programas de melhoramento genético, tem sido realizada no intuito de obter plantas resistentes ou tolerantes ao frio (HIGA et al., 1997).

A tolerância ao frio, que permite às plantas sobreviverem a condições adversas de baixas temperaturas, é um fenômeno complexo, com diferentes mecanismos envolvidos, que depende das espécies envolvidas (LEBORGNE et al., 1995). Para Larcher (2000), muitas plantas apresentam a capacidade de desenvolver mecanismos específicos para a sobrevivência em ambientes que apresentam períodos episódicos e prolongados de congelamento. Ainda, segundo este autor, os mecanismos de evasão e proteção para evitar a formação de gelo nos tecidos definem a capacidade de tolerância e de sobrevivência de uma planta sob um clima frio. A resistência ou tolerância ao frio pode ser uma característica genética que as plantas possuem, bem como pode ser uma característica induzida por modificações ambientais que antecedem as primeiras geadas.

Segundo Levitt (1980) e Nielsen e Orcutt (1996), a tolerância ao frio em plantas podem ser aumentadas através da indução ambiental, causada por exposição a temperaturas baixas, acima do ponto de congelamento. De acordo com esses autores, a indução pode ocorrer quando a planta é exposta durante certo período a temperaturas entre 0 e $10^{\circ} \mathrm{C}$, que é denominado aclimatação ou rustificação. Jacobsen et al. (2007) demonstraram, em quinoa (Chenopodium quinoa Willd.), que o aumento no 
nível de carboidratos solúveis totais nas folhas, em resposta à rustificação das plantas, implica em maior tolerância ao frio.

Para a região Sul do Brasil, Eucalyptus dunnii e Eucalyptus benthamii mostraram-se promissoras para plantios desta espécie em regiões com ocorrência de geadas (EMBRAPA, 1988). O Eucalyptus benthamii apresenta umbom crescimento e resistência a geadas em plantios experimentais de 2 e 3 anos no Estado de Santa Catarina (HIGA et al., 2003). Em áreas de ocorrência de geadas, o Eucalyptus dunnii mostrou boa adaptação, enquanto Eucalyptus grandis e Eucalyptus saligna apresentam sobrevivência inferior a $30 \%$ no oitavo ano de idade, mostrando baixo potencial para plantio nessas regiões (HIGA et al., 1997).

O objetivo deste trabalho foi avaliar o efeito da rustificação sobre a tolerância ao frio em espécies de Eucalyptus, bem como comparar os teores foliares de carboidratos solúveis totais e prolina nestas espécies e verificar suas possíveis relações com a tolerância ao frio.

\section{MATERIAL E MÉTODOS}

\section{Material experimental, local de execução e tratamentos utilizados}

O trabalho foi conduzido com mudas de Eucalyptus dunnii (clone KF 09), Eucalyptus benthamii (clone ARV 116), Eucalyptus saligna (clone 2864) e Eucalyptus grandis (clone 360), com quatro meses de idade, procedentes do viveiro florestal da empresa Klabin, localizado no município de Telêmaco Borba, PR. As mudas de Eucalyptus dunnii (clone KF 09), Eucalyptus benthamii (clone ARV 116) e Eucalyptus saligna (clone 2864) foram obtidas através de sementes procedentes de pomar clonal, localizado em Telêmaco Borba, PR, e as mudas de Eucalyptus grandis (clone 360) foram obtidas de sementes procedentes de Guaíba, RS.

As sementes foram semeadas em tubetes com volume de $90 \mathrm{~cm}^{3}$, contendo substrato constituído de partes iguais de casca de arroz carbonizada e vermiculita tipo 3 , sendo fertilizadas com 4,0 kg de osmocote (19-06-10) + 1,5 kg de superfosfato simples $+500 \mathrm{~g}$ de MAP (monoamônio fosfato) $+150 \mathrm{~g}$ de sulfato ferroso $+150 \mathrm{~g}$ de FTE BR 12 (frited trace elements - micronutrientes) por metro cúbico.

As mudas permaneceram por três meses após a emergência no viveiro da empresa, até atingirem $30 \mathrm{~cm}$ de altura. Em seguida, as mudas foram transplantadas para sacos plásticos com volume de $2000 \mathrm{~cm}^{3}$, contendo o mesmo substrato utilizado na semeadura, permanecendo por mais dois meses no viveiro. Ao final deste período, as mudas atingiram $50 \mathrm{~cm}$ de altura e foram transferidas para o Laboratório de Fisiologia Vegetal do Centro de Ciências Agroveterinárias da Universidade do Estado de Santa Catarina - UDESC, em Lages, SC, para a condução do experimento.

Antes das mudas serem submetidas aos tratamentos de rustificação, elas foram mantidas em câmaras de crescimento marca Eletrolab, modelo $112 \mathrm{FC}$ com fotoperíodo controlado de 12 horas, e temperaturas diurnas de $20{ }^{\circ} \mathrm{C}$ e noturnas de $12{ }^{\circ} \mathrm{C}$, durante três dias, para aclimatação. Após, as temperaturas foram alteradas para temperatura diurna de $10^{\circ} \mathrm{C}$ e noturna de $5^{\circ} \mathrm{C}$, durante quatro dias, mantendo-se o fotoperíodo controlado de 12 horas. Posteriormente, as mudas foram submetidas a dois períodos de rustificação (0 e 21 dias de exposição a temperaturas diurnas de $5{ }^{\circ} \mathrm{C}$ e noturnas de $1{ }^{\circ} \mathrm{C}$ ), com fotoperíodo controlado de 12 horas. Ao final de cada período de rustificação, utilizou-se uma câmara de crescimento, Marca Precision low temperature BOD Incubator $815\left(-10^{\circ} \mathrm{C}\right.$ a $\left.50{ }^{\circ} \mathrm{C}\right)$, modelo $n^{\circ}$. FFU20F3AW4 para submeter as mudas a três gradientes de temperatura abaixo de zero $\left(-2{ }^{\circ} \mathrm{C},-5{ }^{\circ} \mathrm{C}\right.$ e $\left.-8{ }^{\circ} \mathrm{C}\right)$, com três horas de exposição em cada gradiente.

As mudas foram avaliadas quanto às concentrações foliares de carboidratos solúveis totais e de prolina, $\mathrm{TL}_{50}$ (temperatura letal, onde ocorre $50 \%$ da condutividade elétrica máxima) e índice de dano pelo frio.

\section{Determinação de carboidratos solúveis totais}

Folhas frescas foram congeladas em nitrogênio líquido e armazenadas a uma temperatura de $-20{ }^{\circ} \mathrm{C}$, para posterior análise do teor de carboidratos solúveis totais.

A extração e a análise da concentração foliar de carboidratos solúveis totais foram realizadas conforme metodologia descrita por Moraga et al. (2006), utilizando-se o método fenol sulfúrico. As folhas de cada amostra, previamente armazenadas, foram maceradas sem a nervura central, com aproximadamente $25 \mathrm{~mL}$ de nitrogênio líquido, em almofariz e pistilo de porcelana. Após maceradas, foi retirada alícota de $0,3 \mathrm{~g}$ de massa seca, a qual foi colocada em tubo de ensaio, onde se adicionou $3 \mathrm{~mL}$ de etanol a $80 \%$, seguido de agitação. Os tubos de ensaio foram colocados por 30 minutos em banho- 
maria, a uma temperatura de $60^{\circ} \mathrm{C}$. Posteriormente, a amostra foi agitada manualmente, por 30 segundos, e depois centrifugada numa temperatura de $\left(4{ }^{\circ} \mathrm{C}\right)$ a $4000 \mathrm{rpm}$, por 30 minutos. Após a centrifugação, $1 \mathrm{~mL}$ da fase líquida foi transferida para outro tubo de ensaio, adicionando-se a este $1 \mathrm{~mL}$ de clorofórmio e $1 \mathrm{~mL}$ de água deionizada, deixando a mistura em repouso por 45 a 60 minutos, obtendose a separação de fases, estando os pigmentos no fundo do tubo e os carboidratos dissolvidos na parte aquosa superior.

Em outro tubo de ensaio, pipetou-se $200 \mu \mathrm{L}$ da fase aquosa obtida e adicionou-se $1,8 \mathrm{~mL}$ de água deionizada, constituíndo a solução 1. Desta solução 1 , foram retirados $500 \mu \mathrm{L}$ e transferidos para outro tubo de ensaio, onde adicionou-se $500 \mu \mathrm{L}$ de fenol $(5 \%)$ e $2,5 \mathrm{~mL}$ de ácido sulfúrico concentrado. Após, agitou-se em vórtex e, em seguida, foi realizada a medição da absorbância da solução em espectrofotômetro, no comprimento de onda de $485 \mathrm{~nm}$. Para obtenção dos valores de concentração de carboidratos solúveis totais utilizou-se a curva de calibração: $\mathrm{y}=0,0081 \mathrm{x}+$ 0,0744 (onde: $y=$ absorbância; $x=$ concentração de carboidratos; $\mathrm{R}^{2}=0,9988$ ).

\section{Determinação de prolina}

Para determinação de prolina foi utilizada $0,25 \mathrm{~g}$ de matéria seca de folhas maceradas em nitrogênio líquido, sem a nervura principal, juntamente com $5 \mathrm{~mL}$ de ácido sulfosalicílico a $3 \%$. Em seguida, a mistura foi centrifugada por 5 minutos a $3000 \mathrm{rpm}$ em temperatura ambiente, sendo após coletado $1 \mathrm{~mL}$ de sobrenadante e transferidos para tubos de ensaio, adicionando-se $1 \mathrm{~mL}$ de ninidrina ácida e $1 \mathrm{~mL}$ de ácido acético glacial. Posteriormente, as amostras foram colocadas em banho-maria por $1 \mathrm{~h}$ a $100{ }^{\circ} \mathrm{C}$. Após esse período, as amostras foram resfriadas em gelo, sendo adicionados em seguida $2 \mathrm{~mL}$ de tolueno e agitado vigorosamente em vórtex, por 30 segundos, para separação das fases (o tolueno extrai a substância cromófora formando um complexo colorido avermelhado). Após a solução atingir a temperatura ambiente, aspirou-se a fase aquosa superior (cromóforo + tolueno), para leitura em espectrofotômetro no comprimento de onda de $520 \mathrm{~nm}$, conforme descrito por Bates et al. (1973). Para obtenção dos valores de concentração de prolina utilizou-se a curva de calibração: $y=0,0959 x$ - 0,2147 (onde: $y=$ absorbância; $x=$ concentração de prolina; $\left.\mathrm{R}^{2}=0,9926\right)$.

\section{Determinação de $\mathbf{T L}_{50}$}

A $\mathrm{TL}_{50}$ (temperatura letal onde ocorre $50 \%$ da morte celular) foi determinada através da condutividade elétrica (CE) do tecido foliar após as plantas serem submetidas a diferentes gradientes de temperatura abaixo de zero e após os tecidos foliares serem submetidos a uma temperatura de $90{ }^{\circ} \mathrm{C}$ por 4 horas. Para a análise da condutividade elétrica foram utilizadas 10 folhas coletadas da região mediana do quarto par de ramos, sendo retirados dois discos do limbo foliar (com $11 \mathrm{~mm}$ de diâmetro) com perfurador de rolha, na posição mediana da folha, sem atingir a nervura principal.

Após, os discos foram colocados em frascos de vidro com $30 \mathrm{~mL}$ de água deionizada, deixandose em repouso por $24 \mathrm{~h}$ em temperatura ambiente. Em seguida foi realizada a leitura da condutividade elétrica (CE inicial) com condutivímetro Mettler Toledo modelo MPC 227. Após esta leitura, a amostra foi levada para estufa previamente regulada à temperatura de $90^{\circ} \mathrm{C}$, ficando nesta condição por $4 \mathrm{~h}$. Após, a amostra permaneceu em temperatura ambiente por $12 \mathrm{~h}$ e, após este período foi realizada nova leitura da condutividade elétrica (CE final). Através dos valores de CE inicial e CE final, obtémse a $\mathrm{TL}$ através da fórmula $\mathrm{TL}=(\mathrm{CE}$ final-CE inicial $) /(C E$ inicial) $\times 100$, sendo que o gradiente de temperatura abaixo de zero que resulta num valor de $50 \%$ de mortalidade celular é considerado o $\mathrm{TL}_{50}$.

\section{Determinação do índice de dano pelo frio}

$O$ índice de dano pelo frio foi determinado utilizando-se os valores de condutividade elétrica antes e depois de submeter as plantas aos diferentes gradientes de temperatura, e da condutividade elétrica máxima (célula morta), conforme descrito por Flint et al. (1967), de acordo com a fórmula abaixo:

\section{/ CEk) $\} * 100$}

$\mathrm{ID}=\{[(\mathrm{CEf} / \mathrm{CEk})-(\mathrm{CEc} / \mathrm{CEk})] /(1-\mathrm{CEc}$

Em que: ID = Índice de dano; CEf = Condutividade Elétrica do tecido celular após exposição à temperatura abaixo de zero; $\mathrm{CEc}=$ Condutividade Elétrica do tecido celular antes da exposição à temperatura abaixo de zero; CEk $=$ Condutividade Elétrica do tecido celular após exposição à temperatura de $90{ }^{\circ} \mathrm{C}$ por 4 horas (condutividade elétrica máxima).

\section{Delineamento experimental e análise dos dados}

$\mathrm{O}$ experimento foi realizado conforme o delineamento de blocos ao acaso, num esquema 
bifatorial $(2 \times 4)$, com dois períodos de rustificação (0 e 21 dias de exposição das plantas às temperaturas de $5^{\circ} \mathrm{C}$ dia e $1{ }^{\circ} \mathrm{C}$ noite) e quatro espécies (Eucalyptus dunnii, E. benthamii, E. saligna e E. grandis), sendo utilizados três repetições para cada tratamento, com duas plantas por repetição. Também foram avaliados, em cada espécie e nível de rustificação, três gradientes de temperatura abaixo de zero $(-2$, -5 e $-8{ }^{\circ} \mathrm{C}$, por três horas de exposição).

Os dados obtidos foram submetidos à análise da variância, análise de regressão linear (para gradientes de temperatura), análise de correlação por Pearson (para teores foliares de carboidratos solúveis totais, prolina e para temperatura de $\left.\mathrm{TL}_{50}\right)$ e teste de comparação de médias de Tukey $(\mathrm{p}<0,05)$ para níveis de rustificação e espécies de Eucalyptus.

\section{RESULTADOS E DISCUSSÃO}

Nas análises feitas não foi identificada a presença de prolina nos tecidos foliares das espécies avaliadas. No entanto, observou-se diferenças entre as espécies quanto à concentração foliar de carboidratos. Em mudas não rustificadas, o Eucalyptus benthamii apresentou os maiores valores de teores foliares de carboidratos solúveis totais $\left(21,69 \mathrm{mg} \mathrm{g}^{-1}\right)$, seguido do Eucalyptus dunnii (12,95 $\left.\mathrm{mg} \mathrm{g}^{-1}\right)$, que não diferiu do Eucalyptus grandis $\left(12,96 \mathrm{mg} \mathrm{g}^{-1}\right)$ e do Eucalyptus saligna $\left(8,87 \mathrm{mg} \mathrm{g}^{-1}\right)$ (Tabela 1). Todas as espécies apresentaram incremento na concentração foliar de carboidratos solúveis totais com a rustificação (Tabela 1). As maiores concentrações de carboidratos solúveis totais em mudas rustificadas foram observadas em Eucalyptus dunnii $\left(38,15 \mathrm{mg} \mathrm{g}^{-1}\right)$, Eucalyptus benthamii $\left(53,87 \mathrm{mg} \mathrm{g}^{-1}\right)$ e Eucalyptus saligna $\left(25,17 \mathrm{mg} \mathrm{g}^{-1}\right)$, enquanto Eucalyptus grandis apresentou as menores concentrações $\left(16,93 \mathrm{mg} \mathrm{g}^{-1}\right)$. A rustificação causou um aumento na concentração foliar de carboidratos de 2,$9 ; 2,5$; 2,8 e 1,3 vezes em Eucalyptus dunnii, Eucalyptus benthamii, Eucalyptus saligna e Eucalyptus grandis, respectivamente (Tabela 1). O incremento no conteúdo foliar de carboidratos solúveis totais com o aumento no tempo de rustificação ao frio também foi reportado em plantas de cevada (BRAVO et al., 1998) e quinoa (JACOBSEN et al., 2007).

As temperaturas de $\mathrm{TL}_{50}$ nas mudas que não foram submetidas à rustificação, foram mais baixas para o Eucalyptus benthamii $\left(-4,56{ }^{\circ} \mathrm{C}\right)$, seguido por Eucalyptus dunnii $\left(-3,87^{\circ} \mathrm{C}\right)$, sendo maiores em Eucalyptus grandis $\left(-2,00{ }^{\circ} \mathrm{C}\right)$ e Eucalyptus saligna $\left(-2,00{ }^{\circ} \mathrm{C}\right)$ (Tabela 1). Segundo observações a campo, Eucalyptus benthamii e Eucalyptus dunnii apresentam maior resistência ao frio do que Eucalyptus grandis e Eucalyptus saligna (HIGA et al., 1997), estando de acordo com os resultados obtidos no presente trabalho. No entanto, com a rustificação, as quatro espécies avaliadas não diferiram entre si quanto à temperatura de $\mathrm{TL}_{50}$, após 21 dias de rustificação, com valores de $-5,08^{\circ} \mathrm{C},-5,03{ }^{\circ} \mathrm{C},-4,72{ }^{\circ} \mathrm{Ce}-5,02^{\circ} \mathrm{C}$ para Eucalyptus benthamii, Eucalyptus dunnii, Eucalyptus grandis e Eucalyptus saligna, respectivamente (Tabela 1).

Com base neste resultado, e no fato de que no campo, mesmo com a diminuição gradativa das temperaturas antes da ocorrência das primeiras geadas, estas espécies possuem diferentes tolerâncias a baixas temperaturas, podese supor que a rustificação, que ocorre no campo, não é suficiente para conferir tolerância ao frio nas espécies Eucalyptus grandis e Eucalyptus saligna,

TABELA 1: Concentração foliar de carboidratos solúveis totais $\left(\mathrm{mg} \mathrm{g}^{-1}\right)$ e temperatura letal de $50 \%$ $\left(\mathrm{TL}_{50} ;{ }^{\circ} \mathrm{C}\right)$ em quatro espécies de Eucalyptus submetidas a dois períodos de rustificação.

TABLE 1: Leaf concentration of total soluble carbohydrates $\left(\mathrm{mg} \mathrm{g}^{-1}\right)$ and lethal temperature of $50 \%$ $\left(\mathrm{LT}_{50} ;{ }^{\circ} \mathrm{C}\right)$ in four species of Eucalyptus submitted to two acclimatization periods.

\begin{tabular}{|c|c|c|c|c|c|}
\hline \multirow{3}{*}{ Espécies } & \multicolumn{3}{|c|}{ Concentração foliar de carboidratos solúveis $\left(\mathrm{mg} \mathrm{g}^{-1}\right)$} & \multirow{2}{*}{\multicolumn{2}{|c|}{$\begin{array}{c}\mathrm{TL}_{50}\left({ }^{\circ} \mathrm{C}\right) \\
\text { Tempo de rustificação (dias) }\end{array}$}} \\
\hline & \multicolumn{2}{|c|}{ Tempo de rustificação (dias) } & \multirow{2}{*}{$\begin{array}{l}\text { Incremento } \\
\text { (vezes) }\end{array}$} & & \\
\hline & 0 & 21 & & 0 & 21 \\
\hline Eucalyptus benthamii & $21,69 \mathrm{Ba}$ & $53,87 \mathrm{Aa}$ & 2,5 & $-4,56 \mathrm{Aa}$ & $-5,08 \mathrm{Aa}$ \\
\hline Eucalyptus dunnii & $12,95 \mathrm{Bb}$ & $38,15 \mathrm{Ab}$ & 2,9 & $-3,87 \mathrm{Bb}$ & $-5,03 \mathrm{Aa}$ \\
\hline Eucalyptus grandis & $12,96 \mathrm{Bb}$ & $16,93 \mathrm{Ad}$ & 1,3 & $-2,00 \mathrm{Bc}$ & $-4,72 \mathrm{Aa}$ \\
\hline Eucalyptus saligna & $8,87 \mathrm{Bc}$ & $25,17 \mathrm{Ac}$ & 2,8 & $-2,00 \mathrm{Bc}$ & $-5,02 \mathrm{Aa}$ \\
\hline CV (\%) & \multicolumn{3}{|c|}{4,29} & \multicolumn{2}{|c|}{5,57} \\
\hline
\end{tabular}

Em que: médias seguidas pela mesma letra, maiúsculas na horizontal e minúsculas na vertical, não diferem entre si pelo teste de Tukey $(\mathrm{p}<0,05)$. 
ou então a rustificação pode ser influenciada por algum outro fator ambiental.

Observou-se também que a temperatura de $\mathrm{TL}_{50}$ diminuiu com a rustificação, exceto no Eucalyptus benthamii, que não apresentou diferenças significativas entre 0 e 21 dias de rustificação (Tabela 1). Levitt (1980) e Nilsen e Orcutt (1996) também observaram maior tolerância ao frio $\left(\right.$ menor $\left.\mathrm{TL}_{50}\right)$ em plantas expostas à rustificação em temperaturas entre 0 e $10^{\circ} \mathrm{C}$. No entanto, embora o Eucalyptus benthamii tenha apresentado a menor temperatura $\mathrm{TL}_{50}$ não houve um aumento significativo na indução da tolerância ao frio com a rustificação. Segundo Larcher (2000), a tolerância ao frio pode ser uma característica genética ou induzida pelo ambiente. Como o Eucalyptus benthamii apresentou uma baixa temperatura $\mathrm{TL}_{50}$ mesmo sem rustificação, talvez a tolerância ao frio nesta espécie seja uma característica controlada geneticamente e pouco influenciada pelo ambiente.

Houve correlação negativa entre a temperatura de $\mathrm{TL}_{50}$ e a concentração foliar de carboidratos solúveis totais dos genótipos de Eucalyptus avaliados (Figura 1), confirmando resultados obtidos por Moraga et al. (2006). Estes autores afirmaram que a concentração foliar de carboidratos solúveis totais apresenta, na maioria das plantas avaliadas, correlação negativa significativa com a temperatura de $\mathrm{TL}_{50}$. Bravo et al. (2001) observaram em Deschampsia antartica e Colobanthus quitensis, duas espécies da Antártida, que o principal mecanismo de resistência destas plantas ao frio foi através do aumento em até sete vezes nos teores foliares de carboidratos solúveis, com consequente redução da temperatura de $\mathrm{TL}_{50}$. Estes autores também sugeriram que a sobrevivência de plantas na Antártida poderia ser em parte explicada pelo acúmulo foliar de algumas substâncias, tais como os carboidratos solúveis.

Considerando os resultados obtidos no presente trabalho e em outros trabalhos (BRAVO et al., 2001; MORAGA et al., 2006; JACOBSEN et al., 2007), a concentração foliar de carboidratos solúveis totais pode ser um bom indicativo da tolerância de plantas a baixas temperaturas.

Para mudas sem rustificação, Eucalyptus grandis e Eucalyptus saligna apresentaram os maiores índices de dano à temperatura de $-2{ }^{\circ} \mathrm{C}$, comparados com Eucalyptus benthamii e Eucalyptus dunnii (Tabela 2; Figura 2). Esses resultados confirmam os resultados obtidos por Higa (1997), que observou baixo potencial de

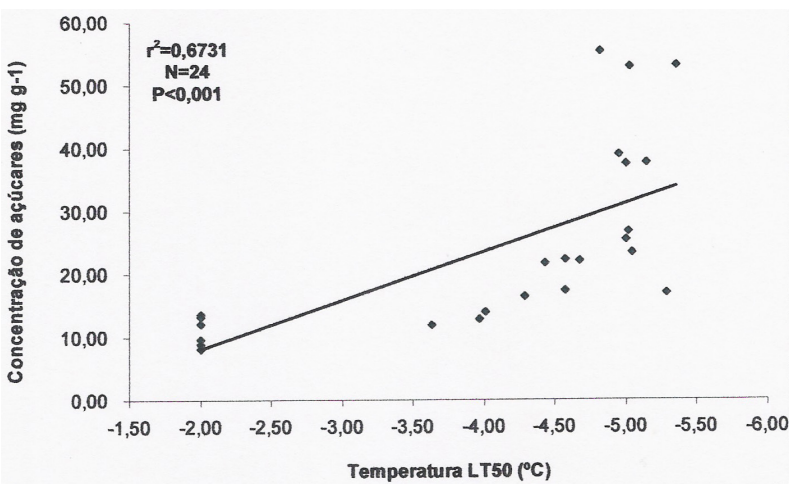

FIGURA 1: Correlação entre temperatura letal de $50 \%\left(\mathrm{TL}_{50} ;{ }^{\circ} \mathrm{C}\right)$ e a concentração de carboidratos solúveis totais $\left(\mathrm{mg} \mathrm{g}^{-1}\right)$ em Eucalyptus dunnii, E. benthamii, E. saligna e E. grandis submetidos a dois períodos de rustificação.

FIGURE 1: Correlation between lethal temperature of $50 \%\left(\mathrm{LT}_{50},{ }^{\circ} \mathrm{C}\right)$ andleafconcentrations of total soluble carbohydrates $\left(\mathrm{mg} \mathrm{g}^{-1}\right)$ in Eucalyptus dunnii, E. benthamii, E. saligna e E. grandis submitted to two acclimatization periods.

TABELA 2: Índice de dano de frio nas espécies de Eucalyptus.

TABLE 2: Index of cold damage of the species of Eucalyptus.

\begin{tabular}{lccc}
\hline \multicolumn{4}{c}{ Indice de dano } \\
\hline Dias de & $-2{ }^{\circ} \mathrm{C}$ & $-5{ }^{\circ} \mathrm{C}$ & $-8{ }^{\circ} \mathrm{C}$ \\
rustificação & \multicolumn{3}{c}{ Eucalyptus benthamii } \\
\hline 0 & $0,04 \mathrm{a}$ & $0,78 \mathrm{a}$ & $0,86 \mathrm{a}$ \\
21 & $0,09 \mathrm{a}$ & $0,46 \mathrm{~b}$ & $0,92 \mathrm{a}$ \\
$\mathrm{CV}(\%)$ & \multicolumn{3}{c}{8,94} \\
\hline \multicolumn{3}{c}{ Eucalyptus dunnii } \\
0 & $0,17 \mathrm{a}$ & $0,92 \mathrm{a}$ & $0,98 \mathrm{a}$ \\
21 & $0,05 \mathrm{a}$ & $0,47 \mathrm{~b}$ & $0,95 \mathrm{a}$ \\
$\mathrm{CV}(\%)$ & \multicolumn{3}{c}{4,38} \\
\hline \multicolumn{3}{c}{ Eucalyptus grandis } \\
\cline { 2 - 4 } 0 & $0,73 \mathrm{a}$ & $0,86 \mathrm{a}$ & $0,88 \mathrm{a}$ \\
21 & $0,15 \mathrm{~b}$ & $0,71 \mathrm{~b}$ & $0,85 \mathrm{a}$ \\
$\mathrm{CV}(\%)$ & \multicolumn{3}{c}{4,21} \\
\hline \multicolumn{3}{c}{ Eucalyptus saligna } \\
\cline { 2 - 4 } 0 & $0,79 \mathrm{a}$ & $0,78 \mathrm{a}$ & $0,98 \mathrm{a}$ \\
21 & $0,04 \mathrm{~b}$ & $0,48 \mathrm{~b}$ & $0,91 \mathrm{~b}$ \\
$\mathrm{CV}(\%)$ & \multicolumn{3}{c}{2,86} \\
\hline
\end{tabular}

Em que: médias não seguidas pela mesma letra na vertical, em cada espécie, não diferem entre si pelo teste de Tukey $(\mathrm{p}<0,05)$. 

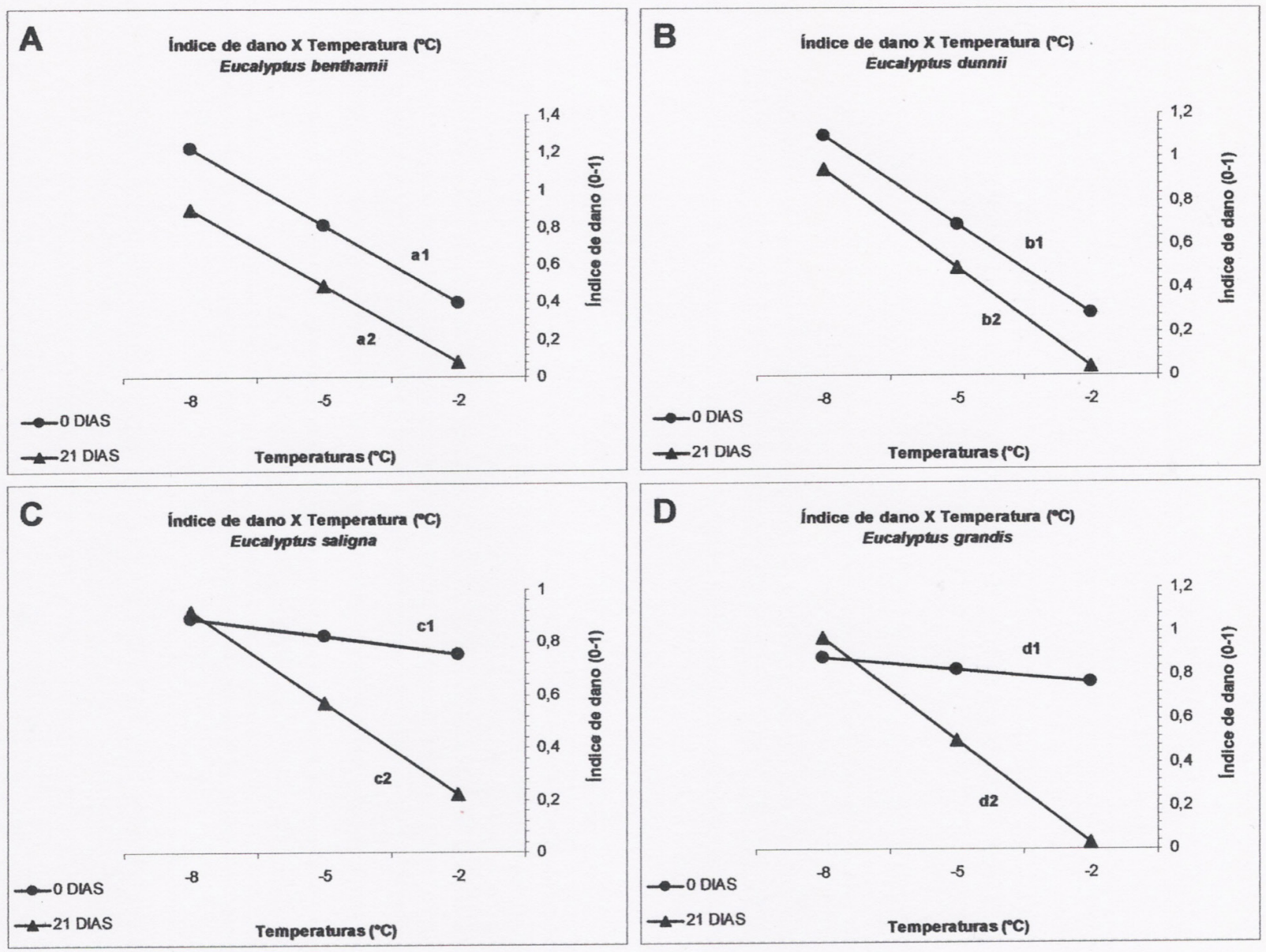

FIGURA 2: Relação do Índice de dano (0-1) em Eucalyptus benthamii (A), Eucalyptus dunnii (B), Eucalyptus saligna (C) e Eucalyptus grandis (D) submetidos a diferentes temperaturas abaixo de zero $\left({ }^{\circ} \mathrm{C}\right)$ e períodos de rustificação ( 0 e 21 dias). ( $\mathrm{y}_{\text {0dias(Eucalvptus.benthamii })}$ a1 $=0,1244-$ $0,1370 \times \mathrm{R}^{2}=0,8286 ; \mathrm{y}_{21 \text { dias(Eucalyptus.benthamii) }} \mathrm{a}=0,1972-0,1372 \mathrm{R}^{2}=0,9954 ; \mathrm{y}_{\text {Odias(Eucalyptus.dunnii) }} \mathrm{b} 1=$ $0,0109-0,1361 \times \mathrm{R}^{2}=0,8067 ; \mathrm{y}_{21 \text { dias(Eucalyptus.dunnii) }} \mathrm{b} 2=-0,2605-0,1506 \mathrm{x} \mathrm{R}^{2}=0,9987 ; \mathrm{y}_{\text {Odias(Eucalyptus. }}$ saligna) $\mathrm{c} 1=0,7102-0,0229 \times \mathrm{R}^{2}=0,8545 ; \mathrm{y}_{21 \text { dias(Eucalyptus.saligna) }} \mathrm{c} 2=-0,0098-0,1161 \times \mathrm{R}^{2}=0,8860$; $\mathrm{y}_{\text {0dias(Eucalyptus.grandis) }} \mathrm{d} 1=0,7265-0,0197 \mathrm{xR}^{2}=0,6656 ; \mathrm{y}_{21 \text { dias(Eucalyptus.grandis })} \mathrm{d} 2=-0,2861-0,1572 \mathrm{x}^{2}=$ 0,9981).

FIGURE 2: Relation of the damage index (0-1) in Eucalyptus benthamii (A), Eucalyptus dunnii (B), Eucalyptus saligna (C) e Eucalyptus grandis (D) submitted to different temperatures below zero $\left({ }^{\circ} \mathrm{C}\right)$ and acclimatization periods ( 0 and 21 days). ( $\mathrm{y}_{\text {Odays(Eucalyptus.benthami })}$ a1 $=0,1244-0,1370 \mathrm{x}$ $\mathrm{R}^{2}=0,8286 ; \mathrm{y}_{21 \text { days (Eucalyptus.benthamii) }}$ a2 $=0,1972-0,1372 \mathrm{x} \mathrm{R}^{2}=0,9954 ; \mathrm{y}_{0 \text { days (Eucalyptus.dunnii) }} \mathrm{b} 1=0,0109-$ $0,1361 \times R^{2}=0,8067 ; y_{21 \text { days (Eucalyptus.dunnii) }} \mathrm{b} 2=-0,2605-0,1506 \times \mathrm{R}^{2}=0,9987 ; \mathrm{y}_{0}$ $\mathrm{c} 1=0,7102-0,0229 \mathrm{x} \mathrm{R}^{2}=0,8545 ; \mathrm{y}_{21}$ days (Eucalyptus.saligna) $\mathrm{c} 2=-0,0098-0,1161 \mathrm{x} \mathrm{R}^{2}=0,8860 ; \mathrm{y}_{0}$ days (Eucalyptus.grandis) $\mathrm{d} 1=0,7265-0,0197 \times \mathrm{R}^{2}=0,6656 ; \mathrm{y}_{21 \text { days (Eucalyptus.grandis) }} \mathrm{d} 2=-0,2861-0,1572 \mathrm{x} \mathrm{R}^{2}=$ 0,9981). 
uso de Eucalyptus grandis e Eucalyptus saligna em regiões de ocorrência de geadas severas. No entanto, o Eucalyptus saligna, quando rustificado num período de 21 dias, com temperaturas diurnas de $5{ }^{\circ} \mathrm{C}$ e noturnas de $1{ }^{\circ} \mathrm{C}$, apresentou maior acúmulo de carboidratos solúveis totais nas folhas, o que aparentemente fez com que o índice de dano nas temperaturas $-2{ }^{\circ} \mathrm{C}$ e $-5{ }^{\circ} \mathrm{C}$ fosse menor $(0,04$ e 0,48 , respectivamente) quando comparado ao índice de dano sem rustificação $(0,79$ e 0,78 , respectivamente), nas mesmas temperaturas. As espécies de Eucalyptus benthamii, Eucalyptus dunnii e Eucalyptus saligna apresentaram em temperaturas de $-5{ }^{\circ} \mathrm{C}$, após rustificação, índices de dano muito próximos $(0,46 ; 0,47$ e 0,48 , respectivamente), mostrando que o acúmulo de carboidratos solúveis totais permitiu maior tolerância ao frio nestas espécies quando rustificadas. Eucalyptus grandis submetidas à temperatura de $-2{ }^{\circ} \mathrm{C}$ após período de rustificação de 21 dias apresentou um índice de dano de 0,15 , mostrando que houve maior tolerância da espécie ao frio quando rustificada. Já na temperatura de $-5{ }^{\circ} \mathrm{C}$, Eucalyptus grandis não apresentou $\mathrm{o}$ mesmo desempenho, mostrando elevado índice de dano ao frio $(0,71)$. Nenhuma das espécies mostrouse tolerante na temperatura de $-8{ }^{\circ} \mathrm{C}$, mesmo após passar pelo período de rustificação (Tabela 2; Figura 2).

$\mathrm{O}$ alto índice de dano a $-2{ }^{\circ} \mathrm{C}$ das espécies de Eucalyptus grandis e Eucalyptus saligna, pode ser resultado de uma intensificação muito rápida do estressor (frio), antes que os mecanismos de defesa fossem ativados, visto que as mesmas não possuem concentrações iniciais de carboidratos solúveis totais (zero dias de rustificação) suficientes para permitir a tolerância ao frio. Porém, quando essas espécies passaram pelo período de rustificação (21 dias), os índices de dano foram muito próximos àqueles de Eucalyptus dunnii e Eucalyptus benthamii. Segundo Larcher (2000), os danos causados pelo congelamento podem ser evitados por mecanismos de proteção e evasão nas células, os quais inibem ou retardam o congelamento do tecido. Um destes processos pode envolver um maior conteúdo de solutos dissolvidos nas células, reduzindo o ponto de congelamento e tornando a célula mais tolerante a baixas temperaturas (JACOBSEN et al., 2007). Para as plantas que devem atravessar com sucesso um período de temperaturas abaixo do ponto de congelamento é essencial que seu protoplasma seja tolerante ao congelamento, sendo essa condição alcançada pela elevada incorporação de fosfolipídios estáveis nas biomembranas e pela acumulação de carboidratos solúveis, polióis, aminoácidos, poliaminas (PALONEN e JUNTTILA, 1999) e também proteínas hidrossolúveis (LARCHER, 2000).

Observa-se que mudas de Eucalyptus benthamii e Eucalyptus dunnii sem rustificação apresentaram valores de índice de dano na temperatura de $-8{ }^{\circ} \mathrm{C}$ estimados pelas equações ajustadas acima dos observados. No entanto, isso pode ocorrer devido ao problema de ajuste nas equações conforme já reportado por Brackmann (2004). Não foram identificadas concentrações de prolina nos tecidos foliares das espécies de Eucalyptus dunnii, Eucalyptus benthamii, Eucalyptus saligna e Eucalyptus grandis (dados não apresentados), como observado por Jacobsen et al. (2007), em quinoa, e Brigas et al. (1989), em brotos e raízes de Juniperus chinensis. No entanto, Swaaij et al. (1985) observaram acúmulo de prolina em brotos de clones de batata expostos em baixa temperatura e estresse hídrico, estando este acúmulo relacionado à tolerância ao frio. Estes autores sugerem que as possíveis funções da prolina seriam osmorregulação e proteção das membranas celulares e das enzimas durante aumento do estresse. Porém, a importância dos altos níveis de prolina para tolerância de plantas ao estresse por baixas temperaturas tem sido questionada, pois o acúmulo poderia ser simplesmente consequência do estresse hídrico (HANSON et al., 1979, HANSON e HITZ, 1982).

Os resultados obtidos mostram que a relação entre a resistência ou tolerância ao frio e a concentração de carboidratos solúveis totais no tecido vegetal pode possibilitar a identificação de genótipos que possam ser cultivados em condições de baixas temperaturas. Além disso, essa característica pode ser incorporada em genótipos através do melhoramento florestal, visando buscar materiais mais adaptados às regiões de frio intenso no sul do Brasil.

\section{CONCLUSÕES}

Os resultados obtidos permitem concluir que:

1) Em Eucalyptus dunnii, Eucalyptus benthamii, Eucalyptus saligna e Eucalyptus grandis existe correlação negativa entre a temperatura de $\mathrm{TL}_{50}$ e a concentração foliar de carboidratos solúveis totais. 
2) A concentração foliar de carboidratos solúveis totais pode ser utilizada como indicador de tolerância ao frio para Eucalyptus dunni (clone KF 09), Eucalyptus benthamii (clone ARV 116), Eucalyptus saligna (clone 2864) e Eucalyptus grandis (clone 360).

3) A concentração foliar de prolina não possui relação com a tolerância ao frio nas quatro espécies de Eucalyptus estudadas.

\section{AGRADECIMENTOS}

Os autores agradecem à empresa Klabin S.A. e a Universidade do Estado de Santa Catarina (UDESC) pela parceria firmada para realização desta pesquisa.

\section{REFERÊNCIAS BIBLIOGRÁFICAS}

BATES, L. S.; WALDRENR, P.; TEARE, I. D. Rapid determination of free proline for water stress studies. Plant and Soil, v. 39, n. 1, p. 205207, 1973.

BRACKMANN, A. et al. Qualidade de Zinnia elegans 'SCARLET' EM SOLUÇÕES CONSERVANTES COM SACAROSE. Revista Brasileira de Agrociências. v. 10, n. 1, p. 127-129, 2004.

BRAVO, L. A. et al. Cold resistance in Antarctic angiosperms. Physiologia Plantarum, v. 111, n. 1, p. 55-65, 2001.

BRAVO, L. A. et al. The role of ABA in freezing tolerance and cold acclimation in barley. Physiologia Plantarum, v. 103, n. 1, p. 17-23, 1998.

BRIGAS, F. J. et al. Influence of photoperiod and temperature on the development of frost tolerance, growth and contents of water, sugars, starch and proline of shoots and roots of juniper (Juniperus chinensis L. 'Pfitrzerana'). Canadian Journal Plant Science, v. 69, n. 1, p. 305-316, 1989.

EMBRAPA. Centro Nacional de Pesquisa de Florestas. (Colombo, PR). Zoneamento ecológico para plantios florestais no estado de Santa Catarina. Colombo, 1988. 113 p. (EMBRAPA-CNPF. Documentos, 21).

FLINT, H. L.; BOYCE, B. R.; BEATTIE, D. J. Index of injury - a useful expression of freezing injury to plant tissues as determined by the electrolytic method. Canadian Journal Plant Science v. 47, n. 2, p. 229-230, 1967.

HANSON, A. D. et al. Capacity for proline accumulation during water stress in barley and its implication for breeding for drought resistance. Crop Science, v. 19, n. 4, p. 489-493, 1979.

HANSON, A. D.; HITZ, W. D. Metabolic responses of mesophytes to Plant water deficits. Plant Physiology, v. 33, n. 6, p. 163-203, 1982.

HIGA, A. R.; GARCIA, C. H.; SANTOS, E. T. Geadas, prejuízos à atividade florestal. Silvicultura. São Paulo, v. 15, n. 58, p. 40-43, 1994.

HIGA, A. R.; HIGA, R. C. V. Indicação de espécies para reflorestamento. In: GALVÃO, A. P. M. (Ed.). Reflorestamento de propriedades rurais para fins produtivos e ambientais: um guia para ações municipais e regionais. Brasília: Embrapa Comunicação para Transferência de Tecnologia, Embrapa Florestas, 2000. p.101-124.

HIGA, R. C. V. et al. Comportamento de vinte espécies de Eucalyptus em área de ocorrência de geadas na região Sul do Brasil. In: IUFRO CONFERENCE ON SILVICULTURE AND IMPROVEMENT OF EUCALYPT, 1997, Salvador. Proceedings... Colombo: EMBRAPA, Centro Nacional de Pesquisa de Florestas, 1997. p.106-110. HIGA, R. C. V.; PEREIRA, J. C. D. Usos Potenciais do Eucalyptus benthamii Maiden et Cambage. Colombo: Embrapa, 2003. (Comunicado Técnico, n. 100).

JACOBSEN, S. E. et al. Frost resistance mechanisms in quinoa (Chenopodium quinoa Willd.). European Journal of Agronomy, v. 26, n. 4, p. 471-475, 2007. LARCHER, W. Ecofisiologia vegetal. São Carlos: Rima, 2000. 398 p.

LEBORGNE, N. et al. Introduction of specific carbohydrates into Eucalyptus gunnii cells increases their freezing tolerance. European Journal of Biochemistry, v. 229, n. 3, p. 710-717, 1995.

LEVITT, J. Responses of plants to environmental stresses: chilling, freezing and higth temperatures stresses. $2^{\text {nd }}$ ed. New York: Academic Press, 1980. p. 67-344.

MORAGA S. P.; ESCOBAR, R.; VALENZUELA, A. S. Resistance to freezing in three Eucalyptus globulus Labill subspecies. Electronic Journal Biotechnology, v. 9, n. 3, p. 310-314, 2006.

NILSEN, E.; ORCUTT, D. The physiology of plants under stress. Incorpo: John Wiley \& Sons, 1996. p.704.

PALONEN, P.; JUNTTILA, O. Cold hardening of raspberry plants in vitro is enhanced by increasing sucrose in the culture medium. Physiologia Plantarum, v. 106, n. 4, p. 386-392, 1999.

PALTA, J. P. et al. Molecular mechanisms of freezethaw injury and cold acclimation in herbaceous plants: 
merging physiological and genetic approaches. freezing stress. NATO AS1 Ser I, v. 16, n. 1, p. 697 NATO AS1, v. 16, n. 16, p.659-680, 1993. 714, 1993.

PALUDZYSZYN FILHO, E.; SANTOS, P. E. T. SWAAIJ, van A. C.; JACOBSEN E.; FEENSTRA, Considerações sobre o plantio de Eucalyptus W. J. Effect of cold hardening, wilting and dunnii no estado do Paraná. Colombo: Embrapa, exogenously applied proline on leaf praline content 2005. (Comunicado Técnico, n. 141). and frost tolerance of several genotypes of Solanum. STEPONKUS, P. L.; UEMURA, M.; WEBB, M. Physiologia Plantarum, v. 64, n. 2, p. 230-236, S. Redesigning crops for increased tolerance to 1985. 